\title{
Self-reported dental treatment needs during the COVID-19 outbreak in Brazil: an infodemiological study
}

\section{Leandro Machado OLIVEIRA ${ }^{(a)}$ \\ Fabrício Batistin ZANATTA(a)}

(a) Universidade Federal de Santa Maria UFSM, Postgraduate Program in Dentistry Department of Stomatology, Santa Maria, RS, Brazil.
Declaration of Interests: The authors certify that they have no commercial or associative interest that represents a conflict of interest in connection with the manuscript.

\section{Corresponding Author:}

Leandro Machado Oliveira

E-mail: leandromachadooliv@gmail.com

https://doi.org/10.1590/1807-3107bor-2020.vol34.01 14

Submitted: June 1, 2020

Accepted for publication: July 16, 2020

Last revision: July 31, 2020
Abstract: The aim of the present infodemiological study was to evaluate whether the COVID-19 outbreak has influenced the volume of content related to the dental treatment needs of Brazilian Twitter users to summarize the trends, and to identify the perceptions of the treatment needed. We collected tweets related to dental care needs of individuals exposed to the COVID-19 outbreak scenario between March 23 to May 4, 2020 and of those not exposed to the COVID-19 pandemic (unexposed group) on the same reported days of 2019 using the terms "dentista (dentist), dente (tooth), siso (third molar), and aparelho (orthodontic appliance)." Descriptive analysis was performed to provide summary statistics of the frequencies of tweets related to different dental treatment needs and also the differences in volume content between the years 2019 and 2020. Moreover, the data were analyzed by qualitative analysis using an inductive approach. A total of 1,763 tweets from 2020 and 1,339 tweets from 2019 were screened. Those tweets posted by non-Brazilian users, duplicates, and those unrelated to dental treatment needs were removed and, therefore 1,197 tweets from 2020 and 719 tweets from 2019 were selected. Content volume related to dental treatment needs greatly increased during the COVID-19 outbreak. Findings from the word cloud and content analysis suggest that dental pain, related or not to the third molar, and problems with orthodontic appliances were the topics most commonly related to dental treatment needs discussed during the COVID-19 outbreak, mainly conveying anxiety and distress. The volume of tweets related to dental treatment needs posted by Brazilian users increased during the COVID-19 outbreak and self-reported pain and urgencies were the most popular topics.

Keywords: COVID-19; Coronavirus; Oral Health; Pandemics; Social Media.

\section{Introduction}

The first case of the coronavirus disease 2019 (COVID-19) was recorded in Brazil on February 25, 2020,126 days after the World Health Organization (WHO) officially declared that the COVID-19 outbreak constitutes a public health emergency of international concern. ${ }^{2}$ Brazil was in second place in the Worldometer ranking on May 24, 2020, presenting 349,113 confirmed cases of COVID-19 and 3,461 tests per one million people. The United States 
was the world leader, presenting 1,666,828 confirmed cases and 43,404 tests per one million people. ${ }^{3}$ Based on those numbers, WHO claimed that Latin America is currently the world's pandemic epicenter and that Brazil is the most affected country as of the time of this writing (June 1, 2020). ${ }^{4}$ Moreover, findings from Imperial College London suggest that the rate of transmission is still above 1 in all states. ${ }^{5}$

In order to restrain the $2019-\mathrm{nCoV}$ infection, preventive measures started to be implemented by state and local governments since the confirmation of the first case, affecting the daily routines of the population. "Physical distancing", the most frequently used measure, aims to reduce contact between people. ${ }^{6,78}$ Such measure has led to the cancellation of many activities and events and has forced people to work from home when applicable. In the context of dental practice, dentists in Brazil were recommended to perform only urgent treatments since March 23, 2020, due to the high risk of cross-infection between dental practitioners and patients. ${ }^{9,10}$

Still, with physical distancing, the psychosocial impact on individuals becomes more severe. In this perspective, the volume of online social interactions increased significantly, ${ }^{11}$ mainly in platforms such as Twitter, which allows us to stay connected even during crises. Logically, COVID-19 has been the major trending topic, with growing numbers turning to social media for information and discussion - which is known as infodemic. An example of a widely discussed topic was the increased risk of contagion in spaces where contact with body fluids occurs, such as dental clinics. ${ }^{12}$ Furthermore, many people have received alarming news about those infected and those who died from the disease, facts that can negatively affect the population and generate stress and fear. ${ }^{13,14}$ All these aspects combined may cause patients to avoid searching for dental treatment, even when they present with urgent needs. Moreover, the psychological burden during the pandemic period may play an important role in the perception of oral health problems.

Recently, a growing body of literature has focused on examining the use of Twitter for public health research. ${ }^{15,16,17}$ A systematic review identified six main uses of this social networking service for public health, and surveillance of public health or diseases is one of the topics described in the taxonomy. ${ }^{18}$ Regarding oral health topics, to the best of our knowledge, only two studies have investigated twitter data for content related to dental treatment needs ${ }^{19,20}$ Based on the current scenario, WHO postulated this line of research as an emerging scientific field and critical area of practice during a pandemic. As there are limited data on oral health during the outbreak, the use of social media can be helpful in screening those patients with dental treatment needs and elaborating strategies during the pandemic and for the post- outbreak period.

This study aimed to evaluate whether the COVID-19 outbreak has influenced the content volume related to the dental treatment needs of Brazilian Twitter users so as to summarize the trends for treatment needs and to identify the users' perception of their needs. We hypothesized that the volume of tweets would be higher during the outbreak period compared to the same six weeks in 2019.

\section{Methodology}

\section{Study design and social media}

This infodemiological time series study was developed to assess the activity of Twitter users in Brazil regarding dental treatment needs during the COVID-19 outbreak. Since federal regulations consider that research using publicly available data does not involve human subjects, this protocol did not require an institutional review board approval from the Human Research Ethics Committee of the Federal University of Santa Maria.

Twitter is an online social media platform where users send and receive short posts (tweets) answering the question "What's happening?" (maximum 280 characters). Retweet is a repost of a tweet. ${ }^{23}$ Twitter currently has 152 million daily users, who produce about 500 million daily tweets. Brazil is ranked sixth on the list of countries with more users, accounting for 14.35 million users in 2019. ${ }^{24}$ The network content is easily accessed, allowing the search for publicly available information.

Besides the significant reach and volume of data, one of the main differences between Twitter and 
other social networks is the wide use of expressions of emotion, which provide unique interactivity between the users thanks to the real-time nature of the content.

\section{Data collection and preprocessing}

We collected tweets on dental care needs between March 23 and May 42020 from individuals exposed to the COVID-19 outbreak scenario (exposed group) and 2019 from those not exposed to the virus (unexposed group) to compare how the outbreak scenario may have influenced the behavior of Twitter users in Brazil and content volume related to their oral health needs.

By means of the Twitter standard search application programming interface (API), we first performed two searches with the terms "dentista" (dentist) and "dente" (tooth) in order to retrieve the maximum number of tweets related to our topic of interest and to identify the most common dental treatment needs. We then generated a word cloud (https://www.wordclouds. com) (Figure 1) and carried out secondary searches based on the most used terms: "siso" (third molar) and "aparelho" (orthodontic appliance).

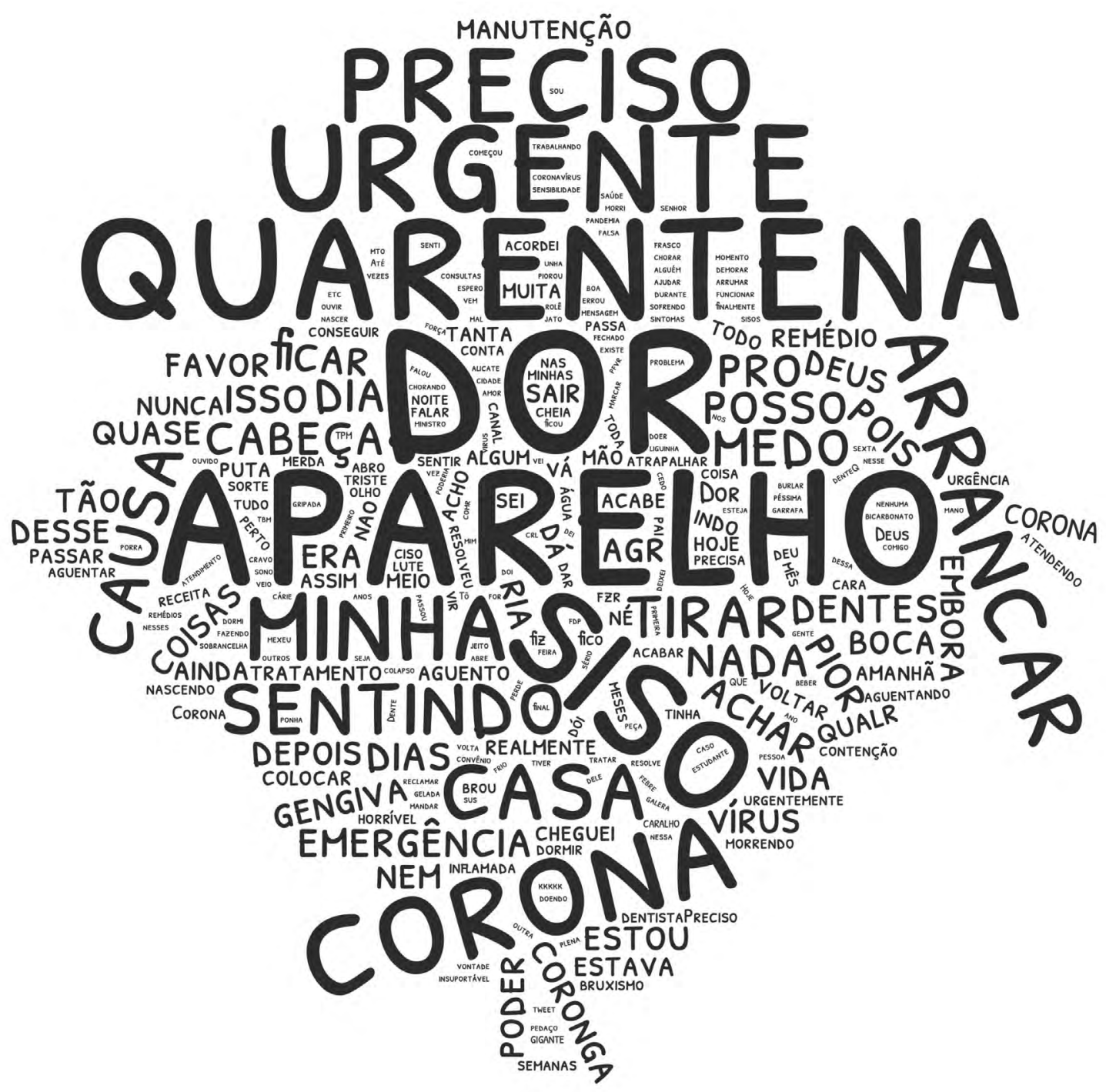

Figure 1. Word cloud created according to the searches with predefined terms. Font size correlates with the greater frequency of the word used in tweets related to self-reported dental treatment needs. 
All the duplicates were removed, and only tweets in Portuguese were collected. We imputed the location of tweets based on Twitter users' selfreported state of residence. As other countries also have Portuguese as their mother tongue, manual searches were performed in Twitter users' profiles in order to distinguish between Brazilian Portuguese and European Portuguese, when location was not informed.

Content was excluded from the dataset of tweets for any of the following reasons: a) content was unrelated to any dental treatment needs or was related to experience of any dental treatment needs; b) content was a subsequent post from the same user (only one post per user); or c) non-Brazilian user.

\section{Data analysis}

We used the R packages twitteR and RCurl to extract and store the text and metadata of the tweets using the time stamp, number of likes and retweets, and user profile information. We stored the tweets in a Microsoft Excel table, where the primary key of the table was tweet content. The included tweets were translated into English by a native Brazilian Portuguese speaker who is also fluent in the English language. Firstly, quantitative analysis was performed following the summative approach of content analysis ${ }^{21,22}$ with the aid of the Stata 14.0 software (StataCorp., College Station, TX, USA), to provide summary statistics for the frequency of tweets on different dental treatment needs and also on the differences in volume content between the years 2019 and 2020. Thereafter, tweets posted in 2020 were analyzed qualitatively using the inductive approach.

\section{Results}

Following the terms of service and privacy policies of Twitter, all data were anonymized and were not reported verbatim to any third party.

\section{Search volume trends}

A total of 1,763 tweets were obtained between March 23 and May 4, 2020 and 1,339 were retrieved from the same days of 2019, totaling 3,102 tweets in the initial screening. Of these tweets, 332 (10.7\%) were from non-Brazilian users, 804 (25.9\%) were unrelated to dental treatment needs or uninterpretable, and $50(1.6 \%)$ were a subsequent post from the same user and were then removed and, therefore, 1,916 tweets (1,197 from 2020 and 719 from 2019) were selected from 1,916 single users. Regarding spatial distribution, only 1,029 (53.7\%) of the analyzed Twitter users provided their locations, and most of them were from the southeastern region $(62.4 \%)$, followed by the southern region (18.7\%), northeastern region $(14.3 \%)$, midwestern region (3.1\%), and northern region $(1.5 \%)$.

Table 1 displays some examples of the extracted tweets retrieved with the predefined search terms and their classifications according to the type of dental treatment needed. The most common reason for excluding a tweet on unrelated topics was memory of experienced pain:

"toothache is certainly the worst pain I ever had" (User \#11)

It is also important to mention that many tweets referred to both pain/urgency and orthodontics simultaneously. When this occurred, the tweet was

Table 1. Content classifications according to the type of dental treatment needed and examples of the extracted tweets retrieved with the predefined search terms.

\begin{tabular}{|c|c|}
\hline Content classifications & Examples \\
\hline Pain & $\begin{array}{l}\text { "Seriously I can't stand this pain in my teeth anymore. I CAN'T STAND IT and the worst thing is that my } \\
\text { appointment with the dentist is only on the } 9^{\text {th }} \text { and until then I'll go on suffering" }\end{array}$ \\
\hline Urgency & "I wish this quarantine would end soon; I need a dentist urgently" \\
\hline Orthodontics & "quarantine should end soon; I need to go to the dentist for a maintenance check-up of my braces" \\
\hline Nonurgent aesthetics & "Damn it, I really need bleaching for these ugly-looking teeth, but I drink coffee like crazy" \\
\hline Undefined & "I need to see a dentist" \\
\hline Unrelated & "all I wish for is a very cold beer to give my teeth pain because of the sensitivity" \\
\hline
\end{tabular}


classified under the pain/urgent category, as in the following examples:

\author{
"I just wish I could go to the dentist soon to get my braces off, \\ I can't stand the pain anymore" (User \#34) \\ "I need to see the dentist urgently for a maintenance check-up \\ of my dental braces" (User \#57)
}

The frequency of tweets content related to self-reported dental treatment needs has increased during the 2019-nCoV pandemic since the first week of analysis, and the largest difference was noted at the end of the fourth week. When the number of retweets was summed with the previous number of tweets, there were 1,408 single users for 2020 and 782 for 2019 (Figure 2).
Table 2 shows the frequency of tweets according to content classifications of the type of self-reported treatment needed during the COVID-19 outbreak. In all of the analyzed weeks, the most common complaint was dental pain and $47 \%$ of the tweets referred to pain in the third molar:

"I can't bear this wisdom tooth pain" (User \#9)

"The wisdom tooth is ruining my Sunday.

I'm stuck at home and, to top it all off, I'm racked with pain..."

(User \#10)

Lastly, the large volume of messages containing terms related to orthodontic appliances/treatment indicates difficulties in dealing with suspended optional dental treatments during the pandemic.

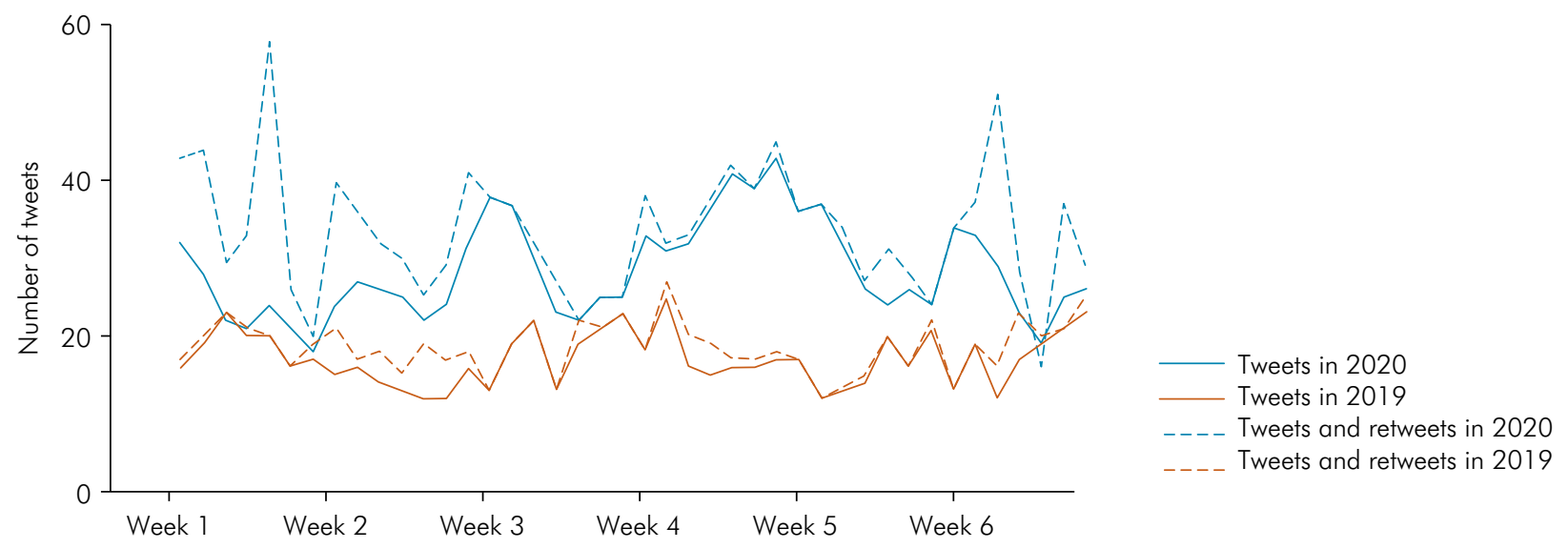

Figure 2. Temporal trends in tweets and retweets related to self-reported dental treatment needs from March 23 to May 4 , 2020 (COVID-19 outbreak) and 2019.

Table 2. Frequency of tweets according to content classifications of the type of self-reported treatment needed during the COVID-19 outbreak.

\begin{tabular}{|c|c|c|c|c|}
\hline \multirow{3}{*}{ Variables } & \multicolumn{4}{|c|}{ Content classifications } \\
\hline & Pain/Urgency & Orthodontics & Nonurgent aesthetics/Undefined & \multirow{2}{*}{ Total } \\
\hline & & n (\%) & & \\
\hline Number of tweets & 881 & 242 & 74 & 1197 \\
\hline Week 1 & $118(73.7)$ & $39(24.4)$ & $3(1.9)$ & 160 \\
\hline Week 2 & $144(79.4)$ & $23(12.2)$ & $15(8.4)$ & 182 \\
\hline Week 3 & $149(75.1)$ & $38(18.8)$ & $13(6.1)$ & 200 \\
\hline Week 4 & $192(74.6)$ & $54(20.7)$ & $13(4.7)$ & 259 \\
\hline Week 5 & $143(69.3)$ & $52(24.9)$ & $13(5.8)$ & 208 \\
\hline Week 6 & $135(72.4)$ & $36(18.9)$ & $17(8.6)$ & 188 \\
\hline
\end{tabular}


"Quarantine, please go away! I need to see my dentist; my braces are getting worn out" (User \#29)

"I'm angry!! My appliance needs to be fixed and I can't go to my dentist because of the corona!" (User \#96)

\section{Main themes}

Based on the users' posts, three main themes and subthemes were identified according to the purpose of the study.

\section{Anxiety and distress}

Most tweets conveyed anxiety and distress over the potential future effects of dental treatment needs. In this topic, the need to see a dentist was considered by many users. Besides, some statements revealed catastrophic association with the current experience, as demonstrate by the following posts:

"I need to see a dentist urgently" (User \#94)

"This toothache is killing me; I need to see my dentist for a maintenance check-up, but dental offices have been closed down for 2 months already..." (User \#213)

"I wish this quarantine would end because I need to go to the dentist; otherwise my teeth will rot with these braces" (User \#594)

Moreover, dental anxiety and the fear of 2019-nCov contagion influenced individuals' decision-making capacity to deal with their dental treatment needs:

"I don't know what is worse: the pain I am feeling or the fear of going to the dentist" (User \#145)

"Ineed to see a dentist, but the fear of the corona is greater" (User\#224)

Distress was also perceived by the users as regret for not having visited the dentist before the outbreak. Moreover, some tweets demonstrated users were bewildered at having dental treatment needs now that the pandemic has set in:

\footnotetext{
"Four months without scheduling a maintenance check-up, and now that I had decided to do it, this corona thing comes up and the dentist's office closes down" (User \#45).
}

"I hadn't had a toothache for 25 years. But then, I happen to have one during a worldwide pandemic, of course." (User \#265)

Impact

Some posts revealed that needs resulting from dental pain impact some aspects or activities in the users' lives. Difficulty sleeping and/or eating and other problems with activities of daily living are present:

\footnotetext{
"I will have to break quarantine to see a dentist tomorrow because I haven't slept for the past weeks, I feel unproductive because of the pain, and I'm already feeling bad. I really don't understand why people break quarantine to go out drinking or to do something useless. It must be conscience..." (User \#8)

"Oh brother, I'm so screwed, I have to have two teeth pulled out because they hurt a lot while I'm eating, and I'll have to put up with this pain every day until corona goes away" (User \#292)
}

Furthermore, some Twitter users had difficulty dealing with suspended optional dental treatments during the pandemic. The posts showed discontent at having to delay treatment:

$$
\begin{aligned}
& \text { "I just wish I could go to the dentist not to delay my treatment } \\
& \text { further. Corona, I hate you!" (User \#633) }
\end{aligned}
$$

"I called up four different dentists, sent messages on Facebook and Instagram, commented on their posts and just nothing happened hahaha. I just want to get my braces off" (User \#702)

\section{Action}

As many users showed difficulty dealing with their dental treatment needs, taking or contemplating an action in response to their needs was also a hotly debated topic. Some users considered performing intemperate actions, as described in the posts:

\footnotetext{
"Quarantine leisure activity: I tried to pull off my braces with a pair of pliers. Result: I need a dentist during the quarantine. Great day." (User \#412)
}

Besides, some users sought advice from dentists on Twitter. However, most respondents were not dental professionals and they would often suggest home remedies as treatments: 
"I have a terrible toothache. An infected wisdom tooth. I can't go to the dental clinic; I don't have a dentist. Can a dentist help me?" (User \#139)

"If you have cloves at home... chewing cloves helps to relieve the toothache" (User \#141)

\section{Discussion}

This study assessed the number and content of tweets on dental treatment needs posted by Brazilian users for six weeks (March 23 to May 4, 2020) during the COVID-19 outbreak. Content analysis demonstrated a progressive increase in the volume of tweets related to self-perceived dental treatment needs when compared to the same six weeks in 2019 and compared to the first week analyzed in 2020. Moreover, in tweets from 2020, users revealed anxiety and distress over their dental treatment needs and reported impairment of some aspects of their lives in addition to describing some actions to deal with their oral health problems.

Physical distancing per se may generate high levels of anxiety and stress, which could lead to greater exposure to social media. In this context, psychological burden can explain the large volume of posts. Nonetheless, three explanatory hypotheses may be formulated for the increase in the volume of tweets on dental treatment needs: the first may be related to the fear of contagion, ${ }^{25}$ which may discourage patients from looking for dental care even if they need urgent treatment. COVID-19 information available in social media worldwide increases day by day with updates on the death toll by country, on the risk of infection, and sometimes with alarming and panicladen news. ${ }^{26}$ Such aspect can elucidate the emotional and psychological impact of the COVID-19 pandemic observed in many tweets. States of anxiety and stress, also related to isolation, could reduce the tolerance and resilience to adverse event $\mathrm{s}^{27}$ and, therefore, these individuals could overestimate their problems. This may explain why some individuals classified their oral health problems as "urgencies" even when they were notably painless. Likewise, most users expressed negative feelings and regretted not having visited the dentist before.
The second assumption is related to the economic impact of the COVID-19 outbreak. It is known that the pandemic has caused an unprecedented challenge for all economic sectors ${ }^{28}$ and, in Brazil, the increase in unemployment has been estimated at $50 \%$ to $100 \%$ according to government data. High healthcare costs are leading to significant financial hardships and have contributed to anxiety and panic symptoms. ${ }^{29} \mathrm{In}$ this perspective, even though dentists are attending to urgencies, the great majority of the population cannot afford to pay for that service in a private practice, increasing the demand for treatment in the public health system, underscoring the importance to strengthen it as soon as possible. ${ }^{30}$

While reducing urgent healthcare services can effectively lower the incidence of cross-infection during dental treatments, timely procedures have been delayed for several oral diseases. If an oral emergency is not treated in a timely manner, there could be serious damage to oral and systemic health. ${ }^{31}$ In this respect, the third hypothesis for the increase in the number of tweets on dental treatment needs may be attributable to inefficient teledentistry services. ${ }^{32}$ Such approach, although limited, has several advantages, allowing patients to seek advice on diagnoses and treatments, especially in emergency situations. However, there are some disadvantages like costs, safety, and implications for confidentially and data protection. ${ }^{33}$

In light of these assumptions, the use of social media during the outbreak becomes fundamental and it may remain so in the post-pandemic period as the volume of dental care services has been reduced to preserve social distancing. Typically, Twitter presents a higher volume of shared messages related to what users experience at the time they are posting, suggesting an opportunity for online engagement of dental professionals to assist these users. Furthermore, the sensationalist use of Twitter can be a great challenge for dental public health because of the significant amount of misinformation, non-evidence-based treatments, and fake news ${ }^{34}$, as observed in our searches. Therefore, the presence of dental professionals in this social media platform would be helpful in providing sound evidence and preventing the spread of fake news. 
In fact, our results should encourage dental professionals to stay online, facilitating and supporting accurate knowledge translation into factual, honest, and cooperative language messages, presented in ways that are understood by and accessible to all individuals. The interactivity presented in Twitter must be explored by dentists to increase users' oral health literacy, and expanded to different social networks. Such actions should also counterbalance the high psychosocial impact related to the COVID-19 pandemic with strategic public health communication, aiming to improve psychological well-being. Finally, dental professionals should be able to perform infometric analyses of dental treatment needs, including quality, as well as information demand (i.e., number of Twitter users reporting dental pain), such as search queries or questions posed on social media.

This study has several important limitations. Firstly, our observations were not made from a random population sample but from a sample of Twitter users. Due to high socioeconomic inequality, more than $20 \%$ of the Brazilian population lives in households with no access to the internet ${ }^{35}$ and it is well established that the most economically disadvantaged have a higher incidence of oral diseases. ${ }^{36}$ Further, some age strata may not use Twitter and then were not aggregated in our sample. We cannot discuss prevalence and incidence of dental treatment needs given that the use of Twitter fluctuates day by day and we do not know how many single users were posting when the data were extracted. Although we included only tweets with verbs used in the present or verbs in the past (when it was possible to identify that the dental treatment need occurred during the outbreak period), suggesting a current dental treatment need, some topics might have been overestimated.

It is also important to mention that as genuine human users were not distinguished from automated or bot accounts, some misclassification bias due to ironic posts might have occurred. However, the use of single users rather than overall message volumes in the construction of the surveillance model may minimize the impact of spam-generating automated accounts.

These findings demonstrate that Twitter used as a surveillance tool may be even more helpful during the current pandemic period and may help with the formulation of oral health strategies for the post-pandemic period. Future studies are needed to investigate the implied sentiments in the tweets to unravel the psychological aspects of those patients reporting dental treatment needs.

\section{Conclusion}

This content analysis indicates that the volume of tweets related to dental treatment needs increased during six weeks of the COVID-19 outbreak. Pain/urgencies and orthodontic treatment were the most common needs, conveying mainly anxiety and distress.

\section{References}

1. The Lancet. COVID-19 in Brazil: "So what?". Lancet. 2020 May;395(10235):1461. https://doi.org/10.1016/S0140-6736(20)31095-3

2. Mahase E. China coronavirus: WHO declares international emergency as death toll exceeds 200. BMJ. 2020 Jan;368:m408. https://doi.org/10.1136/bmi.m408

3. Worldometer.Covid-19 Coronavirus pandemic. 2020 [cited 2020 May 24]. Available from: https://www.worldometers.info/coronavirus/?f bclid=IwAR1W664PgdilTqqISHGXhbDUGSCKIxdRP6WeXrpilqZSunxdDLIR_9V-wzk\#countries

4. Burki T. COVID-19 in Latin America. Lancet Infect Dis. 2020 May;20(5):547-8. https://doi.org/10.1016/S1473-3099(20)30303-0

5. Elsland SL, Johns S. Coronavirus 'not yet under control' in Brazil, scientists warn. London: Imperial College London; 2020 May 11 [cited 2020 May 24]. Available from: https://www.imperial.ac.uk/news/197474/coronavirus-under-control-brazil-scientists-warn/

6. Lewnard JA, Lo NC. Scientific and ethical basis for social-distancing interventions against COVID-19. Lancet Infect Dis. 2020 Jun;20(6):631-3. https://doi.org/10.1016/S1473-3099(20)30190-0

7. Wilder-Smith A, Freedman DO. Isolation, quarantine, social distancing and community containment: pivotal role for old-style public health measures in the novel coronavirus (2019-nCoV) outbreak. J Travel Med. 2020 Mar;27(2):taaa020. https://doi.org/10.1093/jtm/taaa020 
8. Sen-Crowe B, McKenney M, Elkbuli A. Social distancing during the COVID-19 pandemic: staying home save lives. Am J Emerg Med. 2020 Jul;38(7):1519-20. https://doi.org/10.1016/i.ajem.2020.03.063

9. Meng L, Hua F, Bian Z. Coronavirus disease 2019 (COVID-19): emerging and future challenges for dental and oral medicine. J Dent Res. 2020 May;99(5):481-7. https://doi.org/10.1177/0022034520914246

10. Izzetti R, Nisi M, Gabriele M, Graziani F. COVID-19 transmission in dental practice: brief review of preventive measures in Italy. J Dent Res. 2020 Aug;99(9):1030-8. https://doi.org/10.1177/0022034520920580

11. Király O, Potenza MN, Stein DJ, King DL, Hodgins DC, Saunders JB, et al. Preventing problematic internet use during the COVID-19 pandemic: consensus guidance. Compr Psychiatry. 2020 Jul;100:152180. https://doi.org/10.1016/i.comppsych.2020.152180

12. Sabino-Silva R, Jardim AC, Siqueira WL. Coronavirus COVID-19 impacts to dentistry and potential salivary diagnosis. Clin Oral Investig. 2020 Apr;24(4):1619-21. https://doi.org/10.1007/s00784-020-03248-x

13. Park HW, Park S, Chong M. Conversations and medical news frames on twitter: infodemiological study on COVID-19 in South Korea. J Med Internet Res. 2020 May;22(5):e18897. https://doi.org/10.2196/18897

14. Lwin MO, Lu J, Sheldenkar A, Schulz PJ, Shin W, Gupta R, et al. Global sentiments surrounding the COVID-19 pandemic on twitter: analysis of twitter trends. JMIR Public Health Surveill. 2020 May;6(2):e19447. https://doi.org/10.2196/19447

15. Doan S, Yang EW, Tilak SS, Li PW, Zisook DS, Torii M. Extracting health-related causality from twitter messages using natural language processing. BMC Med Inform Decis Mak. 2019 Apr;19(S3 Suppl 3):79. https://doi.org/10.1186/s12911-019-0785-0

16. Gohil S, Vuik S, Darzi A. Sentiment analysis of health care tweets: review of the methods used. JMIR Public Health Surveill. 2018 Apr;4(2):e43. https://doi.org/10.2196/publichealth.5789

17. Zhang Z, Ahmed W. A comparison of information sharing behaviours across 379 health conditions on Twitter. Int J Public Health. 2019 Apr;64(3):431-40. https://doi.org/10.1007/s00038-018-1192-5

18. Sinnenberg L, Buttenheim AM, Padrez K, Mancheno C, Ungar L, Merchant RM. Twitter as a tool for health research: a systematic review. Am J Public Health. 2017 Jan;107(1):el-8. https://doi.org/10.2105/AJPH.2016.303512

19. Heaivilin N, Gerbert B, Page JE, Gibbs JL. Public health surveillance of dental pain via Twitter. J Dent Res. 2011 Sep;90(9):1047-51. https://doi.org/10.1177/0022034511415273

20. Aguirre PE, Strieder AP, Lotto M, Oliveira TM, Rios D, Cruvinel AF, et al. Are the Internet users concerned about molar incisor hypomineralization? An infoveillance study. Int J Paediatr Dent. 2020 Jan;30(1):27-34. https://doi.org/10.1111/ipd.12579

21. Hamad EO, Savundranayagam MY, Holmes JD, Kinsella EA, Johnson AM. Toward a mixed-methods research approach to content analysis in the digital age: the combined content-analysis model and its applications to health care twitter feeds. J Med Internet Res. 2016 Mar;18(3):e60. https://doi.org/10.2196/imir.5391

22. Hsieh HF, Shannon SE. Three approaches to qualitative content analysis. Qual Health Res. 2005 Nov; 15(9):1277-88. https://doi.org/10.1177/1049732305276687

23. Twitter.com. Como retweetar. San Francisco: Twitter; 2020 [cited 2020 May 24]. Available from: https://help.twitter.com/pt/using-twitter/how-to-retweet

24. Statista.com. Leading countries based on number of Twitter users as of July 2020. Hamburg: Statista; 2020 [cited 2020 May 24]. Available from: https://www.statista.com/statistics/242606/number-of-active-twitter-users-in-selected-countries/

25. Toniolo M, Negri F, Antonutti M, Masè M, Facchin D. Unpredictable fall of severe emergent cardiovascular diseases hospital admissions during the COVID-19 pandemic: experience of a single large center in Northern Italy. J Am Heart Assoc. 2020 Jul;9(13):e017122. https://doi.org/10.1161/JAHA.120.017122

26. Abd-Alrazaq A, Alhuwail D, Househ M, Hamdi M, Shah Z. Top concerns of tweeters during the COVID-19 pandemic: infoveillance study. J Med Internet Res. 2020 Apr;22(4):e19016. https://doi.org/10.2196/19016

27. Gajofatto A, Donisi V, Busch IM, Gobbin F, Butturini E, Calabrese M, et al. Biopsychosocial model of resilience in young adults with multiple sclerosis (BPS-ARMS): an observational study protocol exploring psychological reactions early after diagnosis. BMJ Open. 2019 Aug;9(8):e030469. https://doi.org/10.1136/bmjopen-2019-030469

28. Nicola M, Alsafi Z, Sohrabi C, Kerwan A, Al-Jabir A, losifidis C, et al. The socio-economic implications of the coronavirus pandemic (COVID-19): A review [Internet]. Int J Surg. 2020 Jun;78:185-93. Available from: https://linkinghub.elsevier.com/retrieve/pii/S1743919120303162 https://doi.org/10.1016/i.ijsu.2020.04.018

29. KFF. What issues will uninsured people face with testing and treatment for COVID-19? San Francisco: Kaiser Family Foundation; 2020 [cited 2020 May 24]. Available from: https://www.kff.org/uninsured/fact-sheet/what-issues-will-uninsured-people-face-withtesting-and-treatment-for-covid-19/

30. Souza CDF, Gois-Santos VT, Correia DS, Martins-Filho PR, Santos VS. The need to strengthen Primary Health Care in Brazil in the context of the COVID-19 pandemic. Braz Oral Res. 2020;34:e047. https://doi.org/10.1590/1807-3107bor-2020.vol34.0047

31. Guo J, Wu H, Xie H. Letter to the editor: How to deal with suspended oral treatment during the COVID-19 epidemic. J Dent Res. 2020 July;99(8):987. ;002203452092016. https://doi.org/10.1177/0022034520920169 
- Self-reported dental treatment needs during the COVID-19 outbreak in Brazil: an infodemiological study

32. Giudice A, Barone S, Muraca D, Averta F, Diodati F, Antonelli A, et al. Can teledentistry improve the monitoring of patients during the Covid-19 dissemination? A descriptive pilot study. Int J Environ Res Public Health. 2020 May;17(10):3399. https://doi.org/10.3390/ijerph17103399

33. Murillo-Escobar MA, Cardoza-Avendaño L, López-Gutiérrez RM, Cruz-Hernández C. A double chaotic layer encryption algorithm for clinical signals in telemedicine. J Med Syst. 2017 Apr;41(4):59. https://doi.org/10.1007/s10916-017-0698-3

34. Vogel L. Viral misinformation threatens public health. CMAJ. 2017 Dec;189(50):E1567-1567. https://doi.org/10.1503/cmaj.109-5536

35. Instituto Brasileiro de Geografia e Estatística - IBGE. Pesquisa Nacional por Amostra de Domicílios - PNAD. Brasília, DF: Instituto Brasileiro de Geografia e Estatística; 2020 [cited 2020 May 24]. Available from: https://www.ibge.gov.br/estatisticas/sociais/ educacao/9127-pesquisa-nacional-por-amostra-de-domicilios. $h t m l$ ? $\nmid=$ destaques

36. Schwendicke F, Dörfer CE, Schlattmann P, Foster Page L, Thomson WM, Paris S. Socioeconomic inequality and caries: a systematic review and meta-analysis. J Dent Res. 2015 Jan;94(1):10-8. https://doi.org/10.1177/0022034514557546 